19 Revue d'histoire du XIXe siècle

Société d'histoire de la révolution de 1848 et des

révolutions du XIXe siècle

25 | 2002

Le temps et les historiens

\title{
Seconde table ronde. Présentation
}

Jean-Claude Caron

URL : http://journals.openedition.org/rh19/429

DOI : 10.4000/rh19.429

ISSN : $1777-5329$

Éditeur

La Société de 1848

Édition imprimée

Date de publication : 1 décembre 2002

Pagination : 139-140

ISSN : 1265-1354

Référence électronique

Jean-Claude Caron, «Seconde table ronde. Présentation », Revue d'histoire du XIXe siècle [En ligne],

25 | 2002, mis en ligne le 29 juin 2005, consulté le 02 mai 2019. URL : http://journals.openedition.org/ rh19/429; DOl : 10.4000/rh19.429

Ce document a été généré automatiquement le 2 mai 2019.

Tous droits réservés 


\title{
Seconde table ronde. Présentation
}

\author{
Jean-Claude Caron
}

1 Le temps des historiens ou regards sur le passé : l'intitulé de cette table ronde ne naviguet-il pas entre le pléonasme et la litote ? Et pourtant, l'interrogation demeure au quotidien dans la pratique de l'historien, comme dans celle du littéraire, ainsi que le confirme la contribution de Damien Zanone: comment appréhender ce passé, ce temps mort que l'historien a l'immense orgueil de vouloir ressusciter, rendre vif aux vivants? Partant de "l'oubli" de Cabet - un oubli qui est une forme de condamnation à mort -, François Fourn évoque, sans fausse pudeur, cette interrogation sur soi, sur sont présent, sur son actualité, à laquelle l'historien se livre trop souvent sans y réfléchir. Pourquoi tel objet d'histoire? Pourquoi telle approche de l'objet, tel traitement méthodologique? Au final, quel changement sur le présent de celui qui se livre à cet exercice parfois déconcertant qu'est l'écriture de l'histoire? Poser des questions, ce n'est pas déjà y répondre: la formule est un peu facile et camoufle ce qui se joue réellement dans l'écriture de l'histoire, quelle qu'en soit la matière. Mais on accordera que si le temps est la matière de cette écriture, alors se mettent inévitablement en branle des enjeux personnels, propres à l'historien, qui recoupent parfois les enjeux historiques, ceux sur lesquels il écrit.

2 Le choix de l'objet “ temps " renvoie immanquablement à la notion de durée, mais aussi à celles de continuité, de rupture, d'immédiateté, ou encore de mémoire, d'oubli, d'amnésie, à travers lesquelles nous évoluons en permanence, passant consciemment ou non d'une perception du temps à une autre. Passé brûlant, tiède ou refroidi, ou passé présent, réactivé, recomposé. À y réfléchir, si la somme des possibles, en termes de méthode, demeure réduite (l'historien, y compris face à des documents ou des sources qu'il invente parfois, est souvent plus frileux quant à l'usage qu'il convient d'en faire), l'interprétation peut, de son côté, ouvrir des perspectives nouvelles, en particulier lorsque la "boîte à outils" des autres sciences humaines ou sociales est mise à contribution. Les différents participants de cette table ronde ont eu à cœur de produire "en direct", sans le secours d'une contribution écrite, qui a été produite dans l'aprèscoup, une réflexion portant à la fois sur la méthode et l'interprétation. Loin d'être discordantes, leurs interventions permettent de vérifier que des rencontres existent 
entre des thèmes, des périodes, des espaces, et parfois des disciplines, différents. Ainsi du rapport passé/présent (et inversement), mais aussi passé/futur; ainsi également, du couple continuité/rupture; ou encore de l'opposition entre temps court et temps long, entre temps " réel " et temps "fictif"; entre temps de l'événement et temps de son récit. Indirectement, enfin, toutes ces contributions nous invitent à réfléchir à la relation existant entre le temps et l'espace : c'est en particulier vrai dans la contribution de JeanMarc Largeaud sur les temps de Waterloo. L'imaginaire du ou des temps - temps court, temps long - qui reconstruit l'événement Waterloo ne peut s'appréhender que dans un cadre spatial lui donnant sa dimension événementielle; mais l'espace dans lequel est pensé l'événement est également au cœur de l'écriture de l'histoire et de ses variations de temporalité. Peut-on comprendre les discours sur Waterloo sans prendre en considération, suivant les époques, le Paris de l'Arc-de-Triomphe, Sedan ou les champs de bataille de la Première Guerre mondiale?

Le temps des mémorialistes pris en charge par Damien Zanone - qui recense les mémoires historiques publiés dans la première moitié du XIX ${ }^{e}$ siècle - est une invitation à penser la différence des discours ou des modèles narratifs, à la fois dans leur contexte de production et dans leur contexte d'utilisation: voilà au moins une confrontation des temporalités où l'histoire se déploie en majesté, mais dans une forte proximité avec le politique. Celui-ci envahit l'espace funèbre lorsque le temps des ultras et le temps des libéraux sont confrontés par Emmanuel Fureix, à l'occasion des funérailles respectives du duc de Berry et du général Foy. Si les premières sont une invitation à regarder vers un passé inscrit dans la continuité avec le présent, dans une durée qui ne craint pas d'en appeler à l'éternité, les secondes prennent en charge la rupture de 1789 et projettent dans un avenir forcément meilleur le présent intermédiaire. Cela confirme l'idée de cultures du temps qui s'opposeraient dans l'espace public, en différentes circonstances, dont les différentes formes de manifestations politiques témoignent. La gloire abordée par Corinne Legoy dans son étude sur la célébration et la louange des Bourbons sous la Restauration font plus que confirmer le propos. Cette contribution, qui démontre au passage la vraie capillarité des disciplines littéraire et historique, produit pourtant un décalage dans l'interprétation, dans la mesure où elle insiste sur l'immédiateté de la louange, condition même de son existence, quand bien même elle serait fictive: son absence de recul - à la différence des mémoires, par exemple - par rapport à l'événement permet à l'auteure d'évoquer un temps affectif aboutissant à un temps mythique, deux concepts dont la pertinence touchera tous ceux qui sont confrontés à des discours ou des représentations hagiographiques longtemps méprisés ou ignorés de ce fait.

Jean-Claude Caron est professeur à l'université de Clermont-Ferrand

\section{RÉSUMÉS}

Les interventions de François Fourn, Emmanuel Fureix, Jean-Marc Largeaud, Corinne Legoy et Damien Zanone visent à démontrer comment, partant du présent, l'historien interroge le passé, 
construit un objet historique et élabore une forme d'écriture de l'histoire. Au cœur de celle-ci, l'objet-temps, décliné sous toutes ses formes.

Second round table

François Fourn, Emmanuel Fureix, Jean-Marc Largeaud, Corinne Legoy and Damien Zanone papers help to understand how the historian, starting from the present, question the past, build a historical subject et draw up his own way of writing history. In the heart of it, lies the "timeobject", from every angle. 